\title{
Two-Dimensional Representation of Action and Arm-Use Sequences in the Presupplementary and Supplementary Motor Areas
}

\author{
Toshi Nakajima, ${ }^{1,2}$ Ryosuke Hosaka, ${ }^{3,4}$ Ichiro Tsuda, ${ }^{5,6}$ Jun Tanji, ${ }^{1,7}$ and Hajime Mushiake ${ }^{1,2}$ \\ ${ }^{1}$ Department of Physiology, Tohoku University School of Medicine, Sendai, 980-8575, Japan, ${ }^{2}$ Core Research for Evolutional Science and Technology, Japan \\ Science and Technology Agency, Kawaguchi, 332-1102, Japan, ${ }^{3}$ Department of Applied Mathematics, Fukuoka University, Fukuoka, 814-0180, Japan, \\ ${ }^{4}$ Laboratory for Dynamics of Emergent Intelligence, RIKEN Brain Science Institute, Wako, 351-0198, Japan, ${ }^{5}$ Research Institute for Electronic Science and \\ ${ }^{6}$ Research Center for Integrative Mathematics, Hokkaido University, Sapporo, 001-0020, Japan, and ${ }^{7}$ Tohoku University Brain Science Center, Sendai, \\ 980-8577, Japan
}

The medial frontal cortex has been thought to be crucially involved in temporal structuring of behavior in monkeys and humans. We examined neuronal activity in the supplementary and presupplementary motor areas of monkeys to investigate how the nervous system deals with the coding of 16 motor sequences resulting from multiple actions involving bilateral use of the arms. We first found in both areas that this behavioral demand resulted in attribute-based representation of individual motor acts, reflecting functional (action) or anatomical (right/left arm) attributes. Actions were frequently represented according to a body-axis-centered reference frame (supination or pronation) regardless of the arm to be used. Moreover, behavioral sequences were primarily represented with respect to the action- or arm-use sequence rather than the sequence of individual movements. We propose that the two-dimensional attribute-based sequence representation provides a robust and efficient means of processing multiple behavioral sequences.

\section{Introduction}

Temporal structuring of multiple actions is one of the major requirements for the achievement of purposeful behaviors (Lashley, 1951; Tanji, 2001; Rosenbaum, 2010). Previous reports have demonstrated that neuronal activity in the medial motor areas of monkeys takes part in sequencing multiple movements performed with a limb or the eyes (Mushiake et al., 1991; Clower and Alexander, 1998; Shima and Tanji, 2000; Berdyyeva and Olson, 2010). Human studies have also indicated critical involvement of these areas in the organization of complex motor sequences (Gerloff et al., 1997; Amador and Fried, 2004). A question arises as to how these cortical areas deal with the temporal sequencing when the combination of motor sequences grows in number by involving multiple actions and body parts. One possible solution for avoiding difficulties in sequencing a large number of motor elements is the use of group-based sequencing. In group-based sequencing, a

Received Feb. 16, 2013; revised July 22, 2013; accepted July 25, 2013.

Author contributions: T.N., J.T., and H.M. designed research; T.N. and R.H. performed research; T.N. and I.T. contributed unpublished reagents/analytic tools; T.N. analyzed data; T.N., J.T., and H.M. wrote the paper.

This work was supported by grants from the Core Research for Evolutional Science and Technology, Japan Science and Technology Agency (T.N., H.M.), and a Grant-in-Aid for Scientific Research on Innovative Areas "Neural Creativity for Communication (Number 4103)" (Grant 24120702) of the Ministry of Education, Culture, Sports, Science, and Technology (T.N., R.H., H.M., I.T.). We thank M. Kurama, Y. Takahashi, and M. Takahashi for technical assistance and animal care. We thank H. Watanabe for mathematical simulation.

The authors declare no competing financial interests.

Correspondence should be addressed to H. Mushiake, Department of Physiology, Tohoku University School of Medicine, 2-1, Seiryo-cho, Aoba-ku, Sendai, 980-8575, Japan. E-mail: hmushiak@med.tohoku.ac.jp.

DOI:10.1523/JNEUROSCI.0855-13.2013

Copyright $\odot 2013$ the authors $\quad 0270-6474 / 13 / 3315533-12 \$ 15.00 / 0$ basis set of sequential attributes are used to construct each specific sequence. These attributes could consist of the sequence of limbs to be used and the sequence of actions. A motor sequence may be encoded in an attribute-based manner, and individual movements to be performed could be decoded later by the combination of multiple attributes. Is it possible for the neural system to arrange the temporal structure of motor events based on common attributes of individual movements rather than based on the individual movements themselves? To address this issue, we designed a behavioral task that required the sequencing of 16 permutations of individual movements containing common attributes in the anatomical and functional domains. The anatomical attribute was the motor effector used (body part), and the functional attribute was the action referenced in relation to the body axis or the manipulation of an object. We found that neuronal activity in the supplementary motor area (SMA) and presupplementary motor area (preSMA) of the Japanese monkey reflected the group-based sequence with respect to actions and effector use rather than the sequence of individual movements. These results suggest that information regarding individual movements is decomposed into functional (action) and anatomical (effector-use) attributes of the individual movements. The neuronal representation of each attribute appears to be sequentially arranged in parallel and then recombined to specify the next movement. This two-dimensional encoding of common attributes (action and effector use) is viewed as producing robust and efficient sequencing of complex motor behavior involving vast combinations of multiple movements. 


\section{Materials and Methods}

Subjects and behavioral task. The experimental subjects were two Japanese monkeys (Macaca fuscata; one male and one female; weighing 5.8$6.5 \mathrm{~kg}$ ). The animals were cared for in accordance with the Guiding Principles for the Care and Use of Laboratory Animals of the National Institutes of Health and the Guidelines for Institutional Animal Care and Use published by our institute. We trained the monkeys to perform a bimanual sequential motor task. During the experiment, each animal sat in a primate chair holding a handle in each hand. For each trial of this task, the animals were required to perform a motor sequence consisting of two motor elements, each of which resulted in turning a handle at a deflection angle of $>5^{\circ}$. One of four possible movements (left forearm supination or pronation, right forearm supination or pronation) was required for each of the two motor elements. The temporal order of the required motor sequence was selected pseudorandomly and altered in blocks of nine trials. Figure $1 A$ illustrates the sequence of events in a trial within an experimental block. The first three trials in a block were performed under visual guidance of two instructional cues (visually guided trials; Fig. 1A, top). The color of the cue indicated left forearm supination (red) or pronation (blue) and right forearm pronation (yellow) or supination (green). For the remaining six trials in the block, the animal performed the movements instructed in the previous visually guided trials with no visual cues (memory-guided trials; Fig. $1 A$, bottom). At the beginning of a trial, the animal was required to place the two handles in neutral positions with its eyes fixated on the central fixation point (FP) on the screen in front. The monkey was required to maintain the handle placement and eye fixation for $1.5 \mathrm{~s}$, during which the instructional cue for the first movement was presented for $0.5 \mathrm{~s}$ in the visually guided trials. Subsequently, the FP dimmed to serve as the first-movement trigger signal (first GO). The animal was required to perform the first movement within the reaction-time limit (1 s), and the handle was returned to the neutral position. After a delay period of $1 \mathrm{~s}$, during which the instructional cue for the second movement was presented for $0.5 \mathrm{~s}$ (in visually guided trials), the animal was given the trigger signal for the second movement. A series of correct movements without a fixation break was rewarded with the delivery of juice $500 \mathrm{~ms}$ later, followed by a $1.5 \mathrm{~s}$ intertrial interval (ITI). During the ITI, the FP was not presented on the screen, and eye fixation was not required. After six memory-guided trials were completed, an auditory signal indicated the end of the current block and the beginning of a new block. A total of 16 two-movement sequences were presented (Fig. 1B). We examined the activity of individual neurons in the 16 permutations of the motor sequences. The focus of the present study was the analysis of the memory-guided trials. Typically, neuronal data collected during 12 memory-guided trials for each series of correct movements were included in the data file.

Reference frames for action representation. The term "action" has been used to indicate various aspects of motor events (Rizzolatti et al., 2001; Hoshi and Tanji, 2006). In the present study, "action" referred specifically to the functional attribute of a movement, whereas "arm use" referred to an anatomical attribute (i.e., left or right forearm). Because each movement was a combination of action and arm use, the task required the animals to perform two-dimensional sequencing in terms of action and arm use. The present study used two reference frames to define an action: (1) a body-symmetric frame (pronation or supination), representing action in terms of movement toward or away from the longitudinal axis of the body (Fig. 1C, top); and (2) an object-manipulative frame (turning a handle to the left or right), representing action with regard to the object to be manipulated (Fig. 1C, bottom). For example, in the body-symmetric reference frame, left-arm and right-arm pronation were grouped together to constitute the action "pronation." In the object-manipulative reference frame, left-arm pronation and right-arm supination were grouped together to constitute the action "rightward turn of the handle," whereas left-arm supination and right-arm pronation constituted the action "leftward turn of the handle." In the following, action is referred to in the body-symmetric or object-manipulative reference frame.

Surgical and recording methods. After 18 months of training, the monkeys performed the memory-guided trials at the correct rate of $>90 \%$.
An acrylic recording chamber and head-fixation bolts were then implanted on the skull of each animal under aseptic conditions using pentobarbital sodium anesthesia $(30 \mathrm{mg} / \mathrm{kg}$, i.m.) with atropine sulfate. Antibiotics and analgesics were used to prevent postsurgical infection and pain. After complete recovery from the surgery, neuronal activity was recorded in the medial part of the frontal cortex using glass-insulated Elgiloy microelectrodes, which were inserted through the dura mater using a hydraulic microdrive (MO-81; Narishige). We confirmed that the chamber had been successfully placed to cover the bilateral SMA and pre-SMA (Fig. 1D) based on physiological criteria established previously (Matsuzaka et al., 1992). Online data collection was performed using a multichannel acquisition processor and spike-sorting software (Plexon). Sorted unit activities were stored with a record of behavioral events on a computer hard drive. Statistical analyses were performed using the software package R (version 2.15.2; http://www.R-project.org/; R Development Core Team). Electromyography was performed using a pair of silver wire electrodes inserted into muscles studied. We recorded the activity of 46 forelimb and axial muscles while recording neuronal activity. Although these muscles exhibited movement-related activity, no consistent changes in activity occurred before the execution of movement. We confirmed that no task-related changes occurred in muscle activity (in forelimb muscles) of the non-instructed arm and that no arm deflection was detectable. Eye position was monitored using an infrared corneal-reflection-monitoring system at $1 \mathrm{kHz}$ (Millennium G200; Matrox).

Statistical modeling to characterize neuronal selectivity. A model-based statistical analysis of neuronal activity was used to characterize neuronal selectivity in terms of common attributes of movements and individual movements. This analysis systematically set up statistical models that represented the distribution of spike counts across all permutations of the first and second movements. Figure 2 shows the three sets of models we considered: the first and second motor-element-selective models $(A)$ and the sequence-selective models $(B)$. As described below, each set was further divided into attribute-coding models and a movement-based coding model. A model consisted of $m$ Gaussian distributions of spike counts with the same variance but different means. A value of 2 , 4, or 16 was assigned to $m$ according to the statistical model chosen.

The first motor-element-selective models consisted of the bodyreferenced action model, the object-referenced action model, the arm model, and the movement model (Fig. $2 A$, top, from left to right). The body-referenced action model assumed two Gaussian distributions of spike counts $(m=2)$ with the same variance but different means according to the body-referenced action (pronation or supination). Similarly, we assumed two Gaussian distributions $(m=2)$ of spike counts for the object-referenced action model (turn left or right) and the arm model (left or right forearm). These three models were categorized as attributecoding models because each model represented a functional or anatomical attribute of individual movements. For the remaining first movement model, we assumed four Gaussian distributions of spike counts $(m=4)$ with respect to the four movements (left forearm supination, left forearm pronation, right forearm pronation, or right forearm supination). This model was categorized as a movement-based coding model. The design of the second element-selective models (Fig. 2A, bottom) was similar to that of the first element-selective models.

The sequence-selective models consisted of four models: (1) the body-referenced action-sequence model; (2) the object-referenced actionsequence model; (3) the arm-sequence model; and (4) the movementbased sequence model (Fig. $2 B$, from left to right). The body-referenced action-sequence models assumed four Gaussian distributions $(m=4)$ of spike counts with the same variance but different means according to the sequences of body-referenced action (pronation-pronation, pronation-supination, supination-pronation, or supination-supination). Similarly, we assumed four Gaussian distributions $(m=4)$ for the object-referenced action-sequence model regarding the direction of handle rotation (turn left-turn left, turn left-turn right, turn right-turn left, and turn right-turn right) and the arm-sequence model (left arm-left arm, left arm-right arm, right arm-left arm, and right arm-right arm). These three models were categorized as attribute-coding models. The movement-based sequence model assumed independent Gaussian dis- 
A
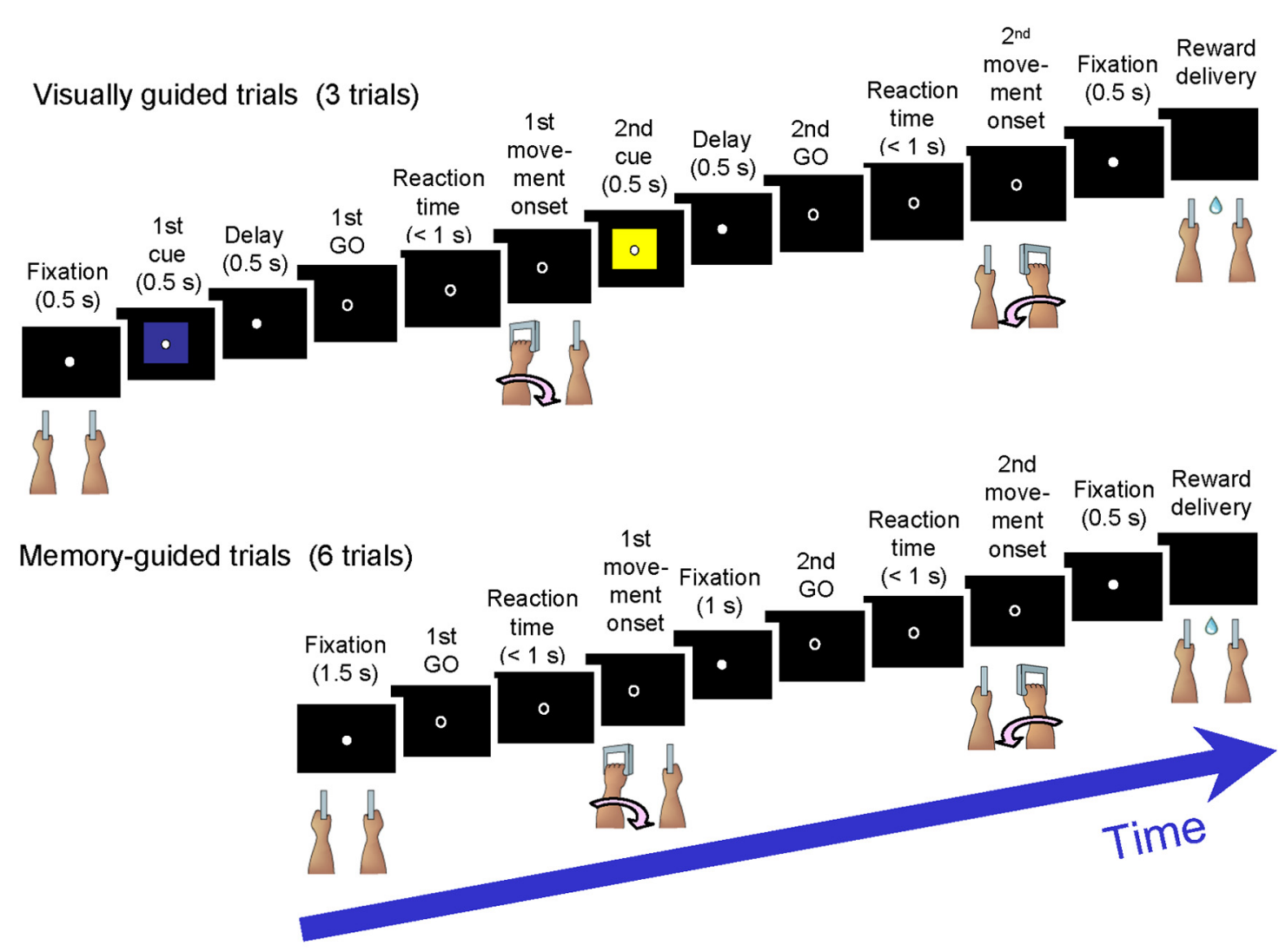

B

\begin{tabular}{|c|c|c|c|c|c|}
\hline & \multicolumn{4}{|c|}{$1^{\text {st }}$ element } \\
\hline & & LS & LP & RP & RS \\
\hline \multirow{4}{*}{ 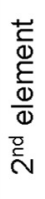 } & LS & LS-LS & LP-LS & RP-LS & RS-LS \\
\hline & LP & LS-LP & LP-LP & RP-LP & RS-LP \\
\hline & RP & LS-RP & LP-RP & RP-RP & RS-RP \\
\hline & RS & LS-RS & LP-RS & RP-RS & RS-RS \\
\hline
\end{tabular}

LS: left forearm supination RS: right forearm supination LP: left forearm pronation RP: right forearm pronation

D

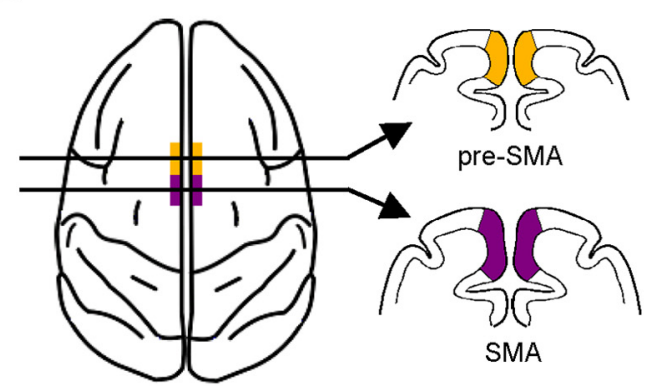

C

Body-symmetric reference frame
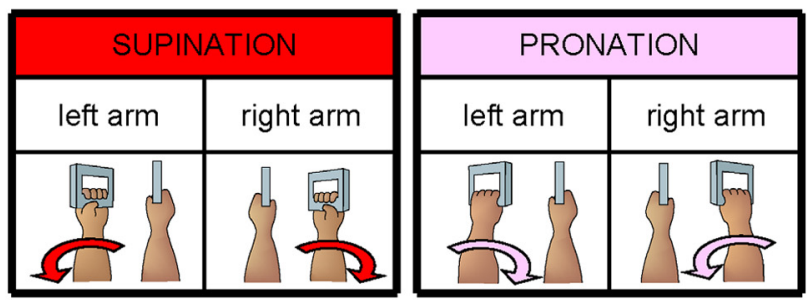

Object-manipulative reference frame

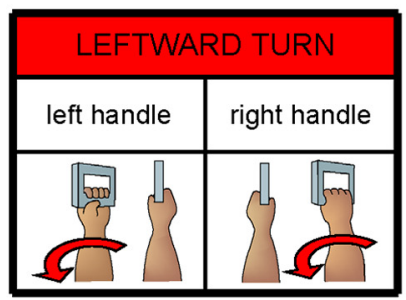

Figure 1. Task sequence, behavioral conditions, and recording sites. $\boldsymbol{A}$, Temporal sequence of events in each experimental block of the behavioral task. Top, Example of a trial in which the animals were required to perform a series of two movements in accordance with two cues (visually guided trial). The monkey was required to memorize a particular motor sequence (left forearm pronation-right forearm pronation in this example) while performing the visually guided trials three times. Bottom, Memory-guided trials in which the order of two movements was memorized; only the GO signals were given. Memory-guided trials of a particular sequence were repeated six times. $\boldsymbol{B}$, A matrix of 16 motor sequences determined the order of pronation or supination of either forearm. $\boldsymbol{C}$, Reference frames for action representation of the behavioral task. Top, The body-symmetric reference frame in which action was defined as the direction of arm rotation (toward or away) with reference to the longitudinal axis of the body. Rotating either forearm away from the body axis was defined as supination (left panel), and rotation toward the body axis was defined as pronation (right panel). Bottom, An object-manipulative reference frame in which action was defined as the direction in which the handle is turned, to the left (left panel) or to the right (right panel). D, Schematic drawing of the recording sites. 


\section{A Activity distribution models representing individual motor elements in the behavioral task}

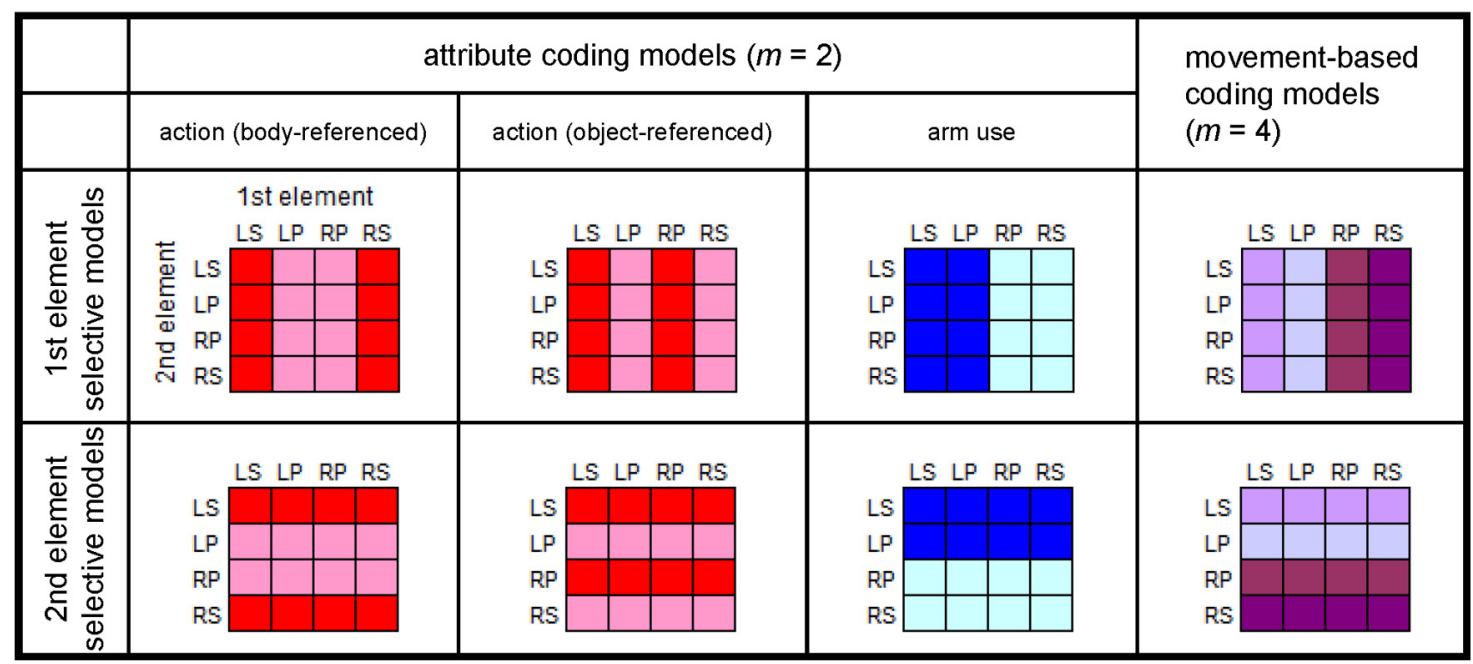

B Activity distribution models representing motor sequences

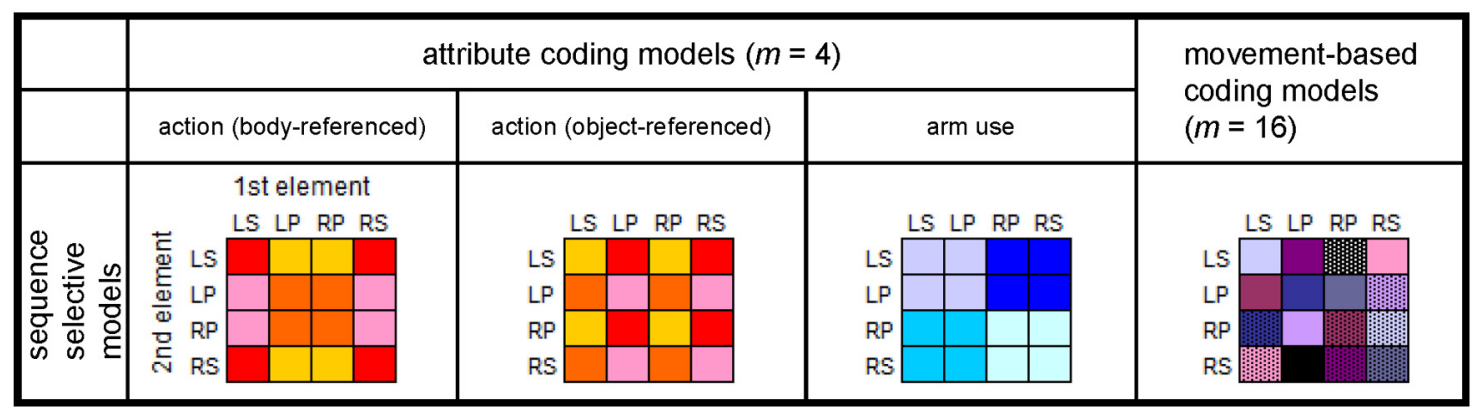

Figure 2. Statistical models representing the distribution of neuronal activity. $\boldsymbol{A}$, Top row, Attribute-coding models (left 3 matrices) and a movement-based coding model (right matrix) defined the selectivity of the first motor element. Each $4 \times 4$ matrix corresponds to a statistical model of activity distribution, in which columns represent the first movement and rows represent the second movement, and each cell in the matrix indicates the magnitude of neuronal activity in a particular motor sequence. For the attribute-coding model, we postulated a bimodal distribution with respect to action or arm use assuming that the distribution within each motor sequence followed a Gaussian distribution with two mean values as indicated by the color code. A quad-modal Gaussian distribution was postulated for the movement-based coding model (movement model). Bottom row, Attribute-coding models (left 3 matrices) and a movement-based coding model (right matrix) defining selectivity for the second motor element. $\boldsymbol{B}$, Attribute-coding models (left 3 matrices) and a movement-based coding model (right) to define the selectivity for motor sequences. The display format is the same as for $A$. We postulated a quad-modal Gaussian distribution $(m=4)$ for each of the three attribute-coding models and a multimodal Gaussian distribution with 16 means $(m=$ 16) for the movement-based coding model (movement-sequence model). LS, Left supination; $R S$, right supination; LP, left pronation; RP, right pronation.

tributions of spike counts with respect to the 16 possible motor sequences $(m=16)$. This model was categorized as a movement-based coding model.

The null hypothesis of no systematic changes in neuronal spike count in a motor element or motor sequence was tested by postulating the null model consisting of a Gaussian distribution of spike counts $(m=1)$. Given a model consisting of $m$ Gaussian distributions, we obtained the set of parameters ( $m$ means and one common variance) that maximized the likelihood of the model and calculated the maximum likelihood $(L)$ by the maximum-likelihood estimation as reported previously (Sakamoto et al., 1986). The number of estimated free parameters for a model was thus $m+1$.

Model evaluation and classification of neuronal activity. We calculated a Bayesian information criterion (BIC) value to measure the goodness of fit of each model to neuronal activity as follows:

$$
\mathrm{BIC}=-2 \log L+(m+1) \log N .
$$

In this formula, $L$ denotes the maximum likelihood of a given model, and $N$ denotes the number of trials (Burnham and Anderson, 2002). The second term penalizes the use of additional free parameters. We selected the model with the smallest BIC value as the best model to characterize the activity of a neuron. Neuronal selectivity was classified according to the best model.
The interval used in the data analysis started $1.5 \mathrm{~s}$ before the onset of the first movement and ended at the onset of the second movement. The time course of neuron selectivity was determined by applying the modelbased statistical analysis to the distribution of spike counts observed in a $200 \mathrm{~ms}$ centered sliding window that stepped across the analytic interval in $20 \mathrm{~ms}$ increments. We defined a neuron as "task related" if its activity matched any statistical model other than the null model for at least three consecutive windows.

A neuron was classified as arm-sequence selective if its activity was armsequence selective for whichever three consecutive windows during the interval analyzed. In this case, the onset time of sequence selectivity was defined as the first instance in which the activity satisfied the criterion. Neurons that were selective for all three sequence types (body-referenced action sequence, object-referenced action sequence, or movement sequence) and their onset times of sequence selectivity were defined in similar ways. If the activity of a neuron corresponded to two or more sequence-selective models at different onset times, it was classified according to the sequence-selective model to which it corresponded in the greatest number of windows. Assuming a Poisson distribution for the spike counts, the aforementioned analysis was performed using raw spike counts and square-root-transformed spike counts (Zar, 2010), and both counts yielded similar results.

To provide support for our BIC-based method of classification, we also performed a post hoc likelihood-ratio test to classify neuronal activity 
into either attribute coding or movement-based coding. This test gave the same classification as BIC did for $>96 \%$ of neuronal data. Thus, we applied BIC-based classification for the present statistical analysis. To have a descriptive measure of assessing how well the best model outperformed the other models, we computed the difference in BIC ( $\Delta \mathrm{BIC})$ between the best model and the second-best model across all the analytic time windows for all the recorded neurons. The median $\triangle \mathrm{BIC}$ resulted in 2.23 for the pre-SMA and 2.19 for the SMA. According to the criteria established by Raftery (1995), both of these values indicate positive or stronger evidence in favor of the best model for a majority of total analytic time windows. The difference of the median $\triangle \mathrm{BIC}$ obtained in the two areas was not significant (Mann-Whitney $U$ test, $W=9.82 \times 10^{9}$, $p=0.16)$.

\section{Results}

Both monkeys performed the behavioral task with a success rate of $>94 \%$ based on memory. The analysis of neuronal activity focused on the memory-guided trials. We analyzed activity of 911 neurons recorded in the pre-SMA and 539 in the SMA. Of these, a total of 891 pre-SMA and 532 SMA neurons were defined as task related and analyzed further. The following section addresses the issue of neuronal selectivity for individual motor elements and, subsequently, selectivity for motor sequences based on the perspectives of attribute coding and movement-based coding.

\section{Selective activity for individual motor elements}

We examined whether neuronal activity preceding the forthcoming motor elements was coded as individual movements or as their common attributes. During the $200 \mathrm{~ms}$ window immediately preceding the onset of the first movement (first premovement period), 306 of 891 (34\%) pre-SMA neurons and 271 of 532 (51\%) SMA neurons were found to be first motor-element selective. Coding the motor element based on an individual movement is the simplest form of motor-element selectivity. An example of such a neuron in the SMA is shown in Figure $3 A$. This neuron was most active before right forearm supination. According to the model-based statistical analysis, this neuron coded individual movements for the first motor element. In contrast, neuronal activity representing either of the two common attributes (i.e., action or arm use) was classified as attribute coding. An example of an attribute-coding neuron in the pre-SMA is shown in Figure $3 B$. Activity was selectively enhanced when the impending first movement was supination, regardless of arm use. This neuron was classified as a neuron encoding the first action. The SMA neuron shown in Figure $3 C$ fired preferentially before using the left arm for the first motor element (regardless of action) and was classified as arm-use selective. In summary, the majority of neurons were attribute coding: $81 \%$ (248 of 306) in the pre-SMA and 69\% (187 of 271) in the SMA (Fig. 3D).

Of the first motor-element-selective neurons, the frequency of action-selective neurons was significantly greater in the pre-SMA (159 of $306,52 \%)$ than in the SMA (92 of 271, 34\%) neurons $\left(p=9.0 \times 10^{-6}\right.$ by Fisher's exact test; Fig. $3 D$, red bars $)$. In contrast, the proportion of movement-selective neurons (movement-based coding neurons indicated by purple bars) was significantly greater in the SMA (84 of 271, 31\%) than in the pre-SMA ( 58 of $306,19 \% ; p=5.6 \times 10^{-4}$ ). The proportion of arm-use-selective neurons (blue bars) was greater in the SMA (35\%) than in the pre-SMA (29\%), although the difference was not significant.

We performed the aforementioned analysis for the neuronal activity during the $200 \mathrm{~ms}$ window immediately preceding the onset of the second movement (second premovement period) and found similar trends in the distribution of coding selectivity for the second motor-element-selective neurons. Overall, 317 of $891(36 \%)$ pre-SMA neurons and 278 of 532 (52\%) SMA neurons were classified as second element selective. An example of an action (pronation)-selective neuron is shown in Figure $4 A$. The majority of second element-selective neurons were attribute coding (Fig. $4 B$ ): $81 \%$ (256 of 317 ) of neurons in the pre-SMA and $64 \%$ (177 of 278 ) of neurons in the SMA. The proportion of action-selective neurons (red bars) was significantly greater in the pre-SMA $(169$ of $317,53 \%)$ than in the SMA ( 85 of $278,31 \%$; $p=$ $3.6 \times 10^{-8}$ by Fisher's exact test). In contrast, the proportion of arm-use-selective neurons (blue bars) was greater in the SMA (33\%) than in the pre-SMA (27\%), although the difference was not significant. The proportion of movement-selective neurons (movement-based coding neurons indicated by purple bars) was significantly greater in the SMA $(101$ of $278,36 \%)$ than in the pre-SMA ( 61 of $317,19 \%$; $\left.p=4.7 \times 10^{-6}\right)$.

\section{Dominance of body-symmetric reference frame for action representation}

Neuronal activity representing action was characterized according to two reference frames for controlling action: (1) bodysymmetric and (2) object-manipulative (Fig. $1 C$ and Materials and Methods). We compared the proportion of neurons coding action in the body-symmetric reference frame and in the objectmanipulative reference frame to determine whether one reference frame was dominant. We found that action was represented primarily in the body-symmetric reference frame. In the preSMA, the majority of neurons (112 of $159,70 \%$ ) were classified as body-referenced action selective, whereas 47 of 159 (30\%) neurons showed selectivity for object-referenced action. The proportion of the neurons selective for body-referenced action was significantly greater than chance (binomial test, $p=1.3 \times 10^{-7}$ ). Furthermore, body-symmetric reference frame dominance was also observed in the SMA ( 55 of 92, 60\%). The same comparison for neuronal activity during the second premovement period yielded similar results.

\section{Sequence-selective activity: two-dimensional sequencing with respect to action and arm use}

The model-based statistical analysis to classify task-related neuronal activity (see Materials and Methods and Fig. 2) revealed that 422 of 891 (47\%) pre-SMA and 260 of 532 (49\%) SMA neurons were motor-sequence selective. The simplest form of motor-sequence selectivity is coding the motor sequence based on individual movements. An example neuron fired preferentially when the first movement was left pronation and when the forthcoming second movement was left supination (Fig. 5A). However, such movement-based coding was found in only a minority of motor-sequence-selective neurons in the pre-SMA (45 of $422,11 \%$ ) and SMA (40 of 260, 15\%) (Fig. 5D, purple bars). More often, the neuronal activity exhibited two types of groupbased sequence selectivity, preferring the sequence with regard to a common attribute of the two intended movements. An example of the first type is shown in Figure $5 B$, where neuronal activity preferentially increased when the first movement was left supination (LS) and the second movement was left or right supination (LS or RS), or when the first movement was right supination (RS) followed by left or right supination (LS or RS). This neuronal activity was deemed selective for the supination-supination sequence (regardless of arm use) and was classified as actionsequence selective. The second type of neuronal activity preferring sequences of a common attribute is shown in Figure $5 C$. Activity was preferentially enhanced if the first movement was to 


\section{A Movement-selective activity}
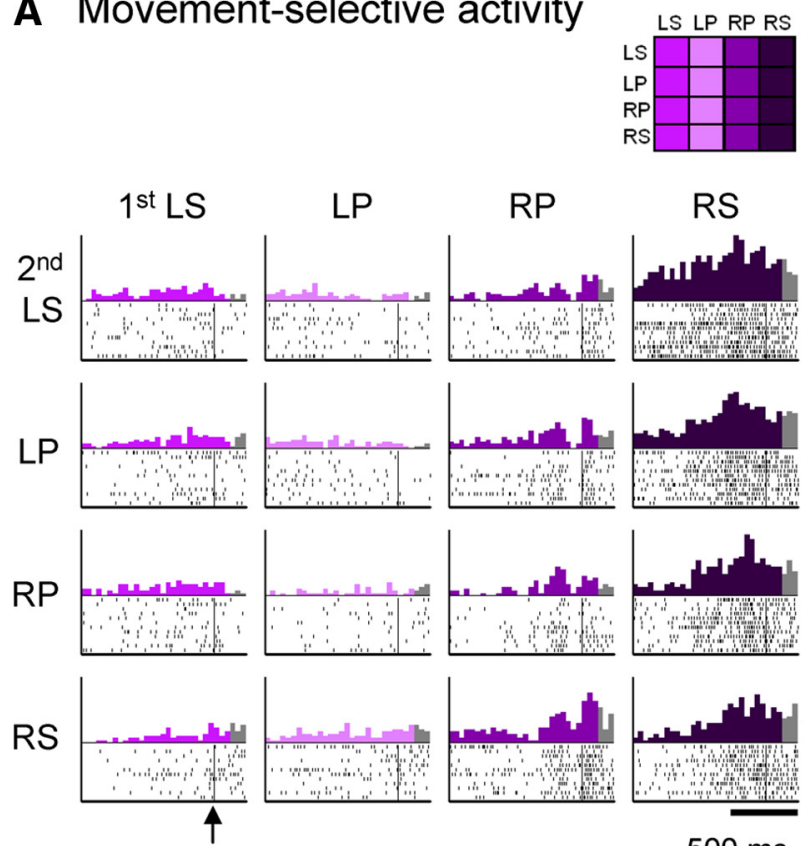

1st movement

onset

\section{Arm-use-selective activity}
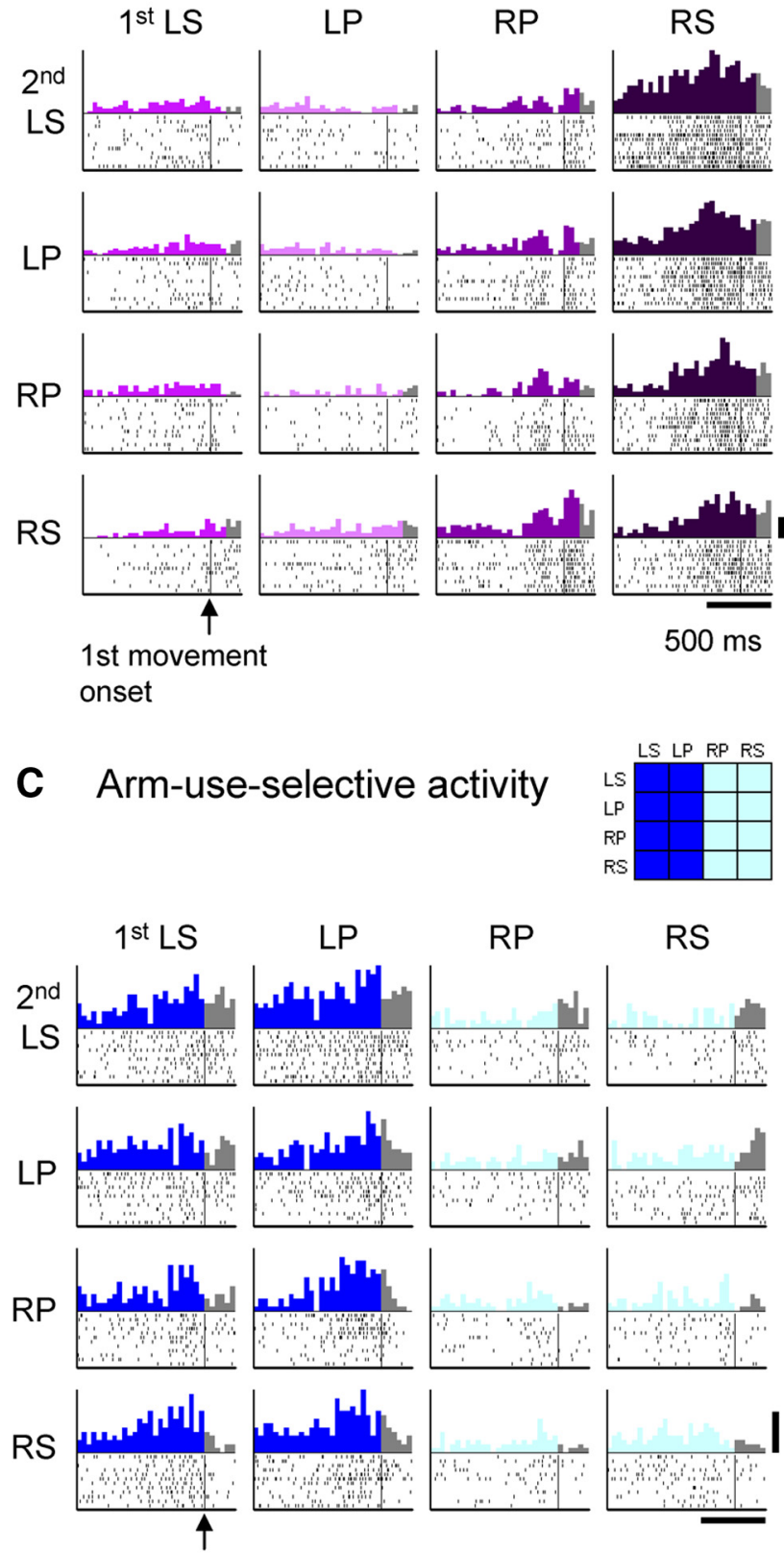

B Action-selective activity
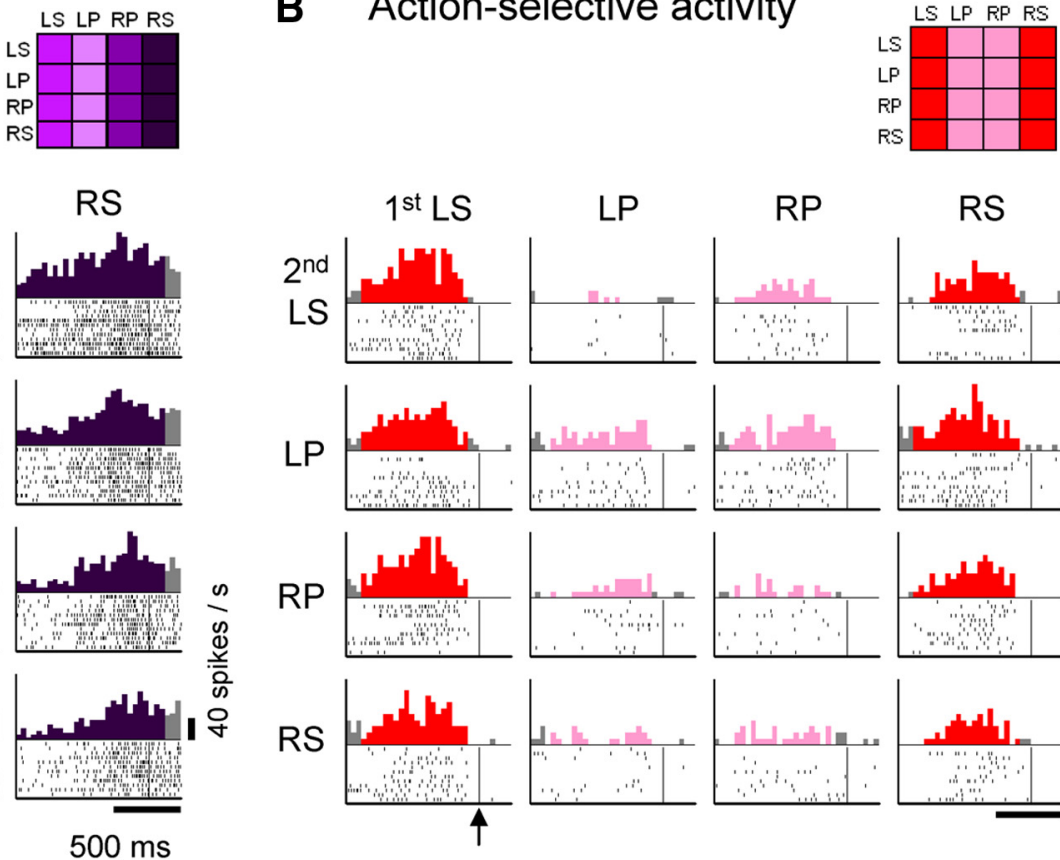

RS
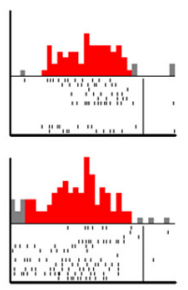

\section{$\frac{\infty}{2}$ 운}

\section{Distribution of 1 st motor-element selective neurons}
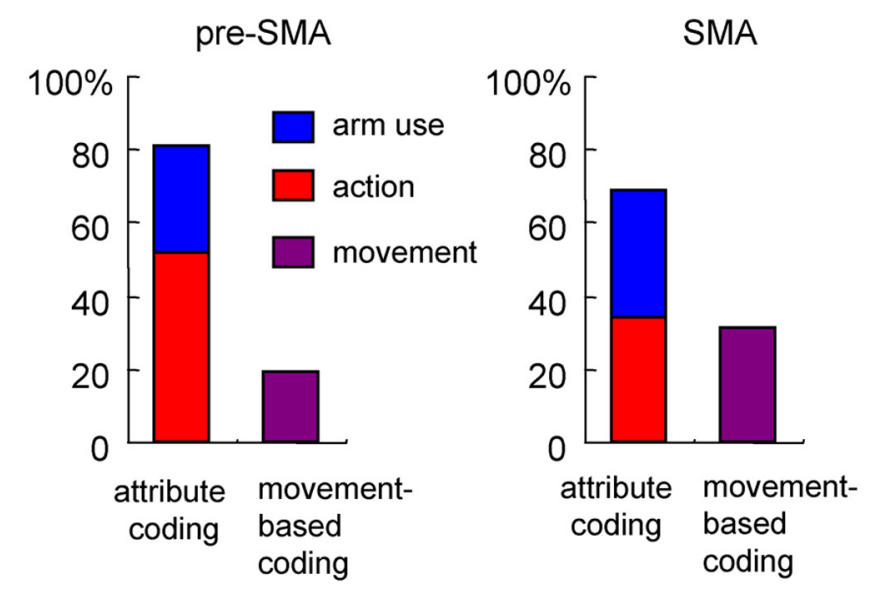

Figure 3. Neuronal activity representing forthcoming movement, action, or arm use. A, An SMA neuron showing enhanced selective activity when right arm supination was the forthcoming first movement in the behavioral task. The rasters show neuronal discharges aligned at the onset of the first movement (vertical lines), and the perievent histograms indicate the sum of discharges with a $40 \mathrm{~ms}$ bin width. Rasters and histograms are displayed in a matrix arranged with respect to the first movement (columns) and second movement (rows). The thick horizontal line at the bottom represents $500 \mathrm{~ms}$. The thick vertical line at the bottom right indicates the 40 spikes/s firing rate. For the time period during which the category of representation of neuronal activity corresponded to the model matrix shown at the top right (for this example, the first movement model), histograms are color coded according to the model matrix. For the remaining period, histograms are colored gray. The display format of the rasters and histograms in $\boldsymbol{B}$ and $\boldsymbol{C}$ is the same as that in $\boldsymbol{A}$. $\boldsymbol{B}$, Action-selective activity in a pre-SMA neuron observed before initiation of the first motor element. In this example, neuronal activity is preferential for the impending selection and initiation of supination, regardless of the laterality of arm use. $\boldsymbol{C}$, Arm-use-selective activity in an SMA neuron. In this example, neuronal activity is preferential for the impending use of the left arm, regardless of whether the selected action is supination or pronation. $D$, Distribution of neurons exhibiting selectivity for the arm-use, action, or individual movements performed as the first motor element in the behavioral task. LS, Left supination; RS, right supination; LP, left pronation; RP, right pronation.

be performed with the right forearm and the second movement involved the left forearm. This type of activity was classified as selective for the arm-use sequence. Overall, 89\% (377 of 422) of pre-SMA and $85 \%$ (220 of 260) of SMA neurons were selective for either the action sequence or arm-use sequence; these were regarded as coding the sequence in terms of motor attributes. The proportion of action-sequence-selective neurons (Fig. $5 D$, red bars) was significantly greater in the pre-SMA (210 of 422, 50\%) than in the SMA ( 99 of 260, 38\%; $p=0.0019$ by Fisher's exact test). In contrast, the proportion of arm-sequence-selective neu- 


\section{A Action-selective activity}

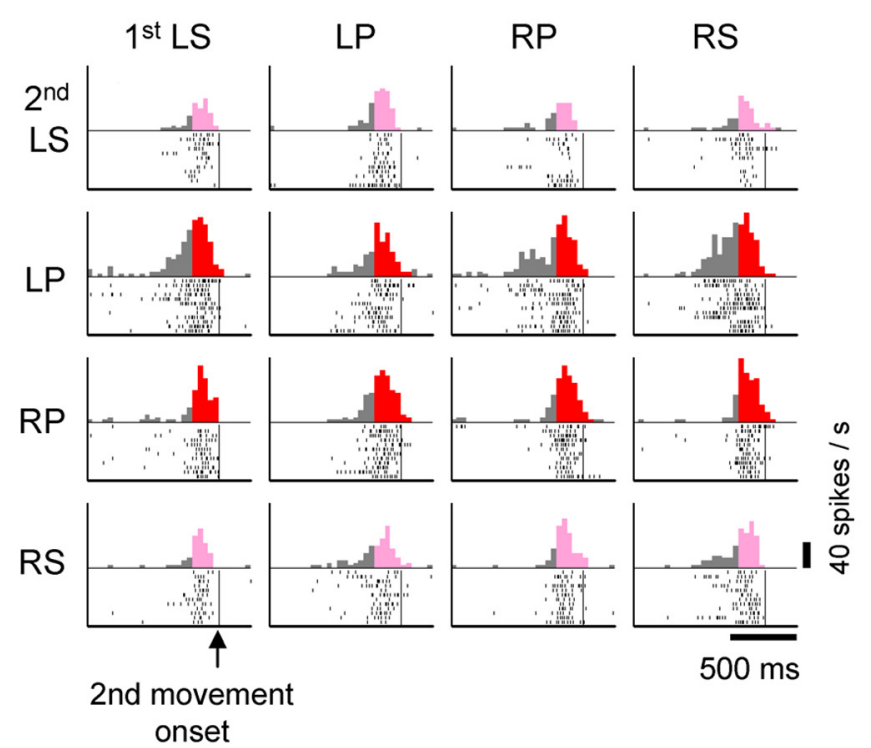

\section{B Distribution of 2nd motor-element selective neurons}

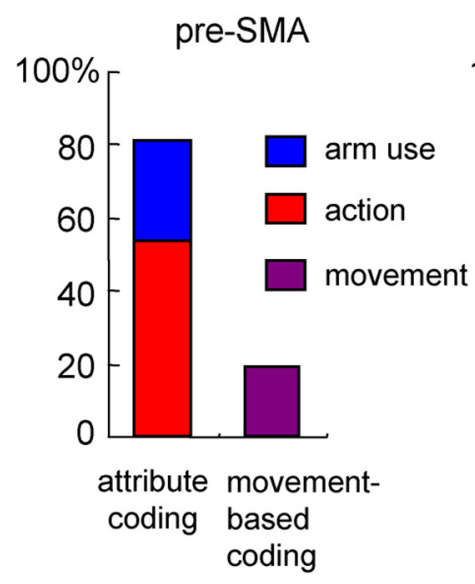

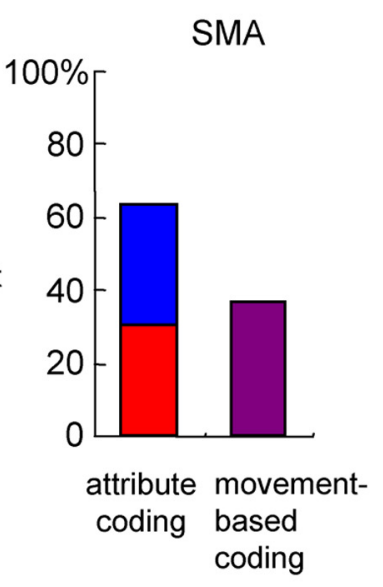

Figure 4. Neuronal activity during the second premovement period. A, Action-selective activity of a pre-SMA neuron observed before initiation of the second motor element in the behavioral task. In this example, the increase in neuronal activity was most pronounced when the impending movement was pronation, regardless of arm laterality. The display format of the rasters and histograms is the same as for Figure 3 except that neuronal activity is aligned with the onset of the second movement (vertical lines). $\boldsymbol{B}$, Distribution of neurons exhibiting selectivity for arm-use, action, or individual movements performed as the second motor element in the behavioral task. LS, Left supination; RS, right supination; LP, left pronation; RP, right pronation.

rons (blue bars) was significantly greater in the SMA (121 of 260, $47 \%$ ) than in the pre-SMA ( 167 of $422,40 \%$; $p=0.044$ ). These results demonstrate that, at the single-neuron level, temporal structuring of movements is formulated along two dimensions with regard to action and arm use and that neurons in the medial motor areas primarily use two-dimensional coding, rather than movement-based coding, to represent multiple motor sequences.

To determine whether the time course of the two types of sequence selectivity differed during the task, we plotted the time of appearance of sequence-selective activity for action-sequenceselective neurons (Fig. 6A) and arm-sequence-selective neurons (Fig. $6 B$ ) relative to the onset of the first and second movements. Although selective activity appeared over a wide range of times in both types, onset of activity selective for action sequence tended to occur earlier than activity selective for arm sequence in the pre-SMA. To test for statistically significant differences in the time course, we compared the percentage of early-onset actionsequence-selective and arm-sequence-selective neurons before the onset of the first movement. In the pre-SMA, 106 of 210 (50.5\%) action-sequence-selective neurons and 66 of 167 (39.5\%) arm-sequence-selective neurons were classified as early onset. The action-sequence-selective neurons included a significantly greater percentage of early-onset neurons than did the arm-sequence-selective neurons (Fisher's exact test, $p=0.022$ ). However, the percentage of early-onset action-sequence-selective neurons (43 of $99,42.4 \%$ ) in the SMA was not significantly different from that of the arm-sequence-selective neurons (47 of $121,38.8 \% ; p=0.29$ ). The onset times of sequence selectivity were examined in greater detail by calculating the cumulative fraction of neurons that exhibited either type of sequence selectivity as a function of time relative to the onset of the first movement (Fig. 6C). In the pre-SMA, selectivity for the action sequence emerged significantly earlier than selectivity for the arm sequence (Kolmogorov-Smirnov test, $D=0.29, p=7.5 \times$ $\left.10^{-6}\right)$. Moreover, the median time for action-sequence selectivity $(-440 \mathrm{~ms})$ was significantly less than that for arm-sequence selectivity ( $60 \mathrm{~ms} ; W=14405, p=4.4 \times 10^{-5}$ by Mann-Whitney $U$ test). A similar trend was observed in the SMA, although the differences were not significant. These results indicate that neural processing of attribute-based sequencing occurs earlier for the functional attribute (action) than for the anatomical attribute (arm use) in the pre-SMA.

\section{Robustness and efficiency of two-dimensional coding}

The predominance of neuronal activity representing action sequence and arm-use sequence raises the question of why twodimensional encoding is more highly favored than movementbased sequence encoding. Considered from the perspective of information representation by a neuronal population, we find relevant literature that tackled the problem with regard to either robustness or efficiency (Rieke, 1996; Simoncelli and Olshausen, 2001; Sreenivasan and Fiete, 2011). We hypothesized that twodimensional coding has advantages from the standpoint of robustness and efficiency in encoding behavioral sequences. To visualize this hypothesis, we constructed two neural network models: (1) a modular network that used two-dimensional coding; and (2) a non-modular network that used movement-based coding (Fig. 7) based on the framework of formal models proposed previously (Louie, 1985; Birkhoff, 1993; Hatakeyama and Tsuda, 2007). The modular network (Fig. 7A) consisted of two independent modules: (1) a module for functional attribute (action module); and (2) one for anatomical attribute (arm-use module). We assumed that each module was equipped with processing units and neural elements; a processing unit specified a particular sequence of either attribute, whereas a neural element represented action or arm use occurring in a sequence. For the 


\section{A Movement-sequence- selective activity \\ (left pronation $\rightarrow$ left supination)}

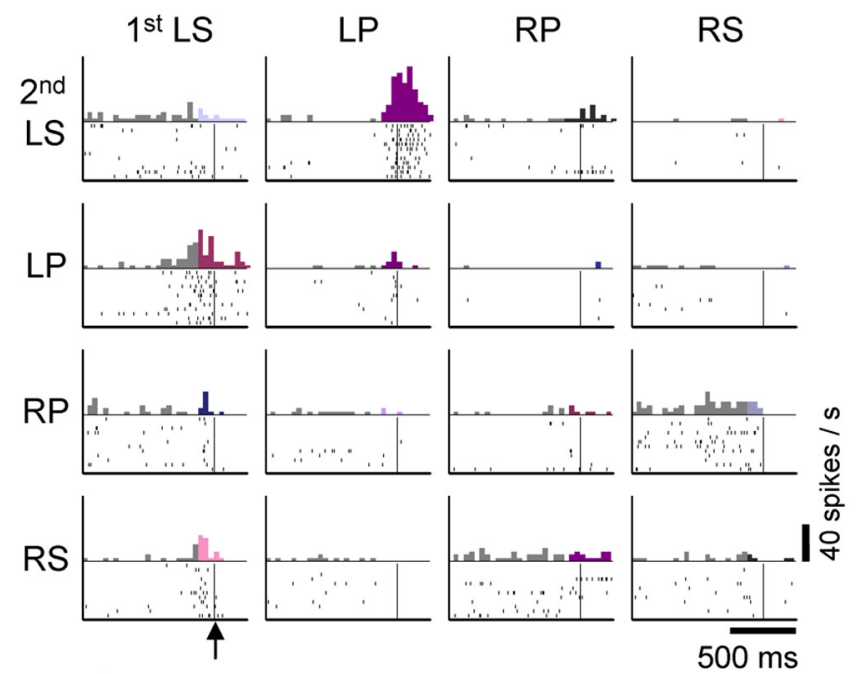

1st movement onset

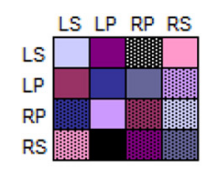

RS

$500 \mathrm{~ms}$

\section{B Action-sequence- selective activity (supination $\rightarrow$ supination)}
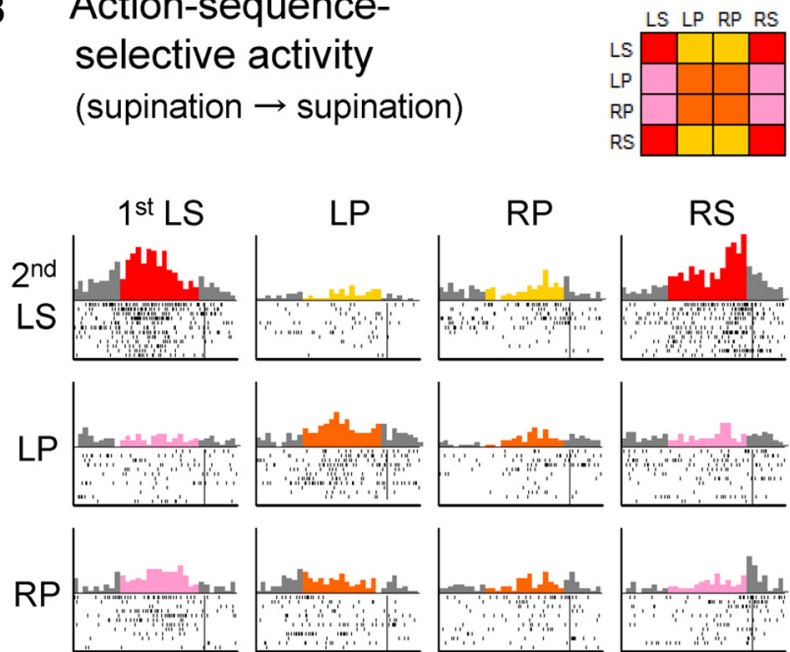

RS
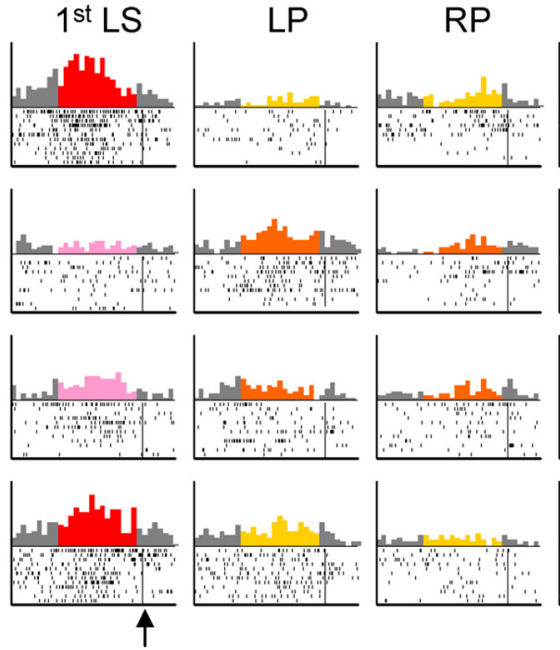

RS
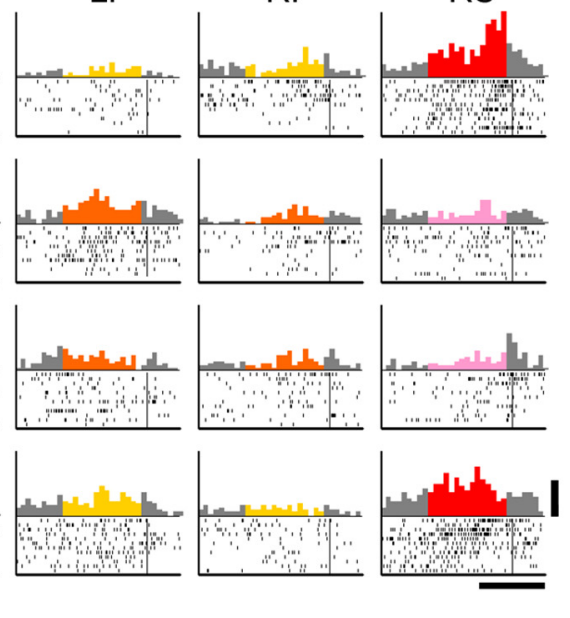

C

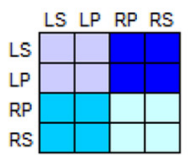

RS

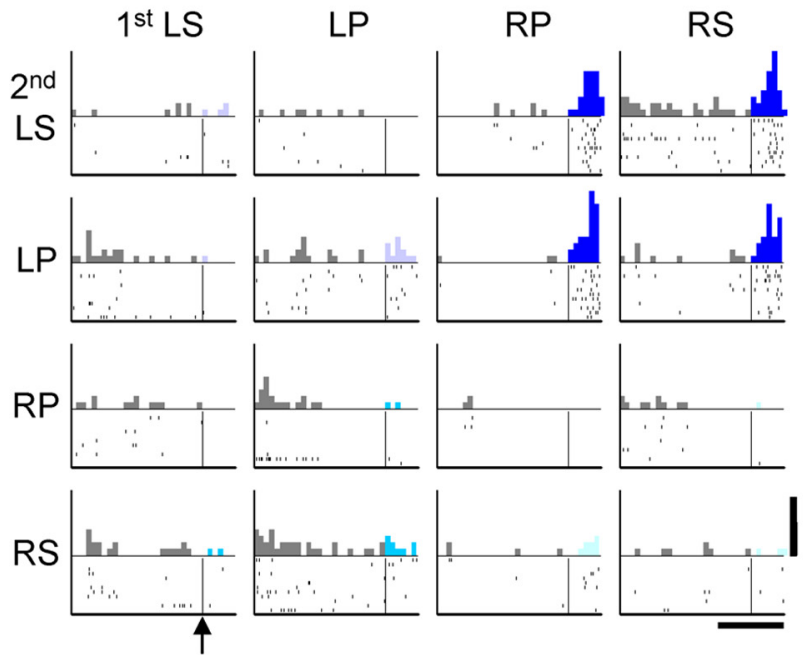

\section{Distribution of motor-sequence selective neurons}
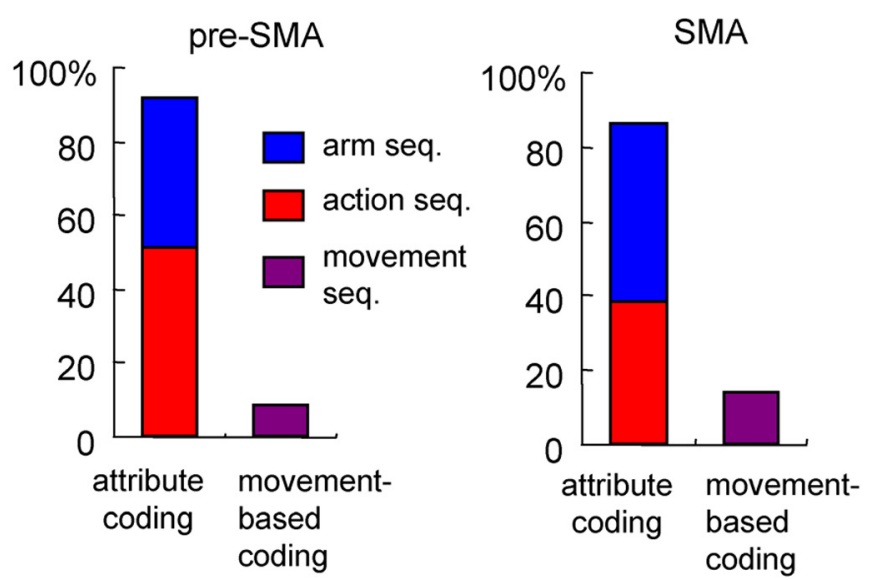

Figure 5. Neuronal activity reflecting three categories of behavioral sequences. The rasters and histograms for each motor sequence are formatted as in Figure $3 . A$, Activity of the pre-SMA neuron preferentially increased when left pronation was followed by left supination, reflecting the sequence of individual movements. $\boldsymbol{B}$, Activity of the pre-SMA neuron preferentially increased when the impending first action was supination only when the second action was also supination, indicating selectivity for the forthcoming sequence of action, regardless of arm use (action-sequence selectivity). C, Pre-SMA neuron exhibiting preferential enhanced activity when the right arm performed the first motor act followed by the left arm for the second motor act, regardless of action (arm-use sequence selectivity). D, Distribution of sequence-selective neurons in the pre-SMA and SMA, exhibiting selectivity for the sequence of arm use, action, and individual movements. LS, Left supination; $R S$, right supination; LP, left pronation; $R P$, right pronation.

non-modular network (Fig. $7 B$ ), we assumed $4 \times 4=16$ processing units, each of which corresponded to a particular sequence of individual movements. Figure 7 shows both networks operating to perform an example motor sequence "left forearm pronationleft forearm supination." We examined the performance of these two networks in terms of robustness and efficiency.

The robustness $(R)$ of a network was quantitatively estimated as the probability that all the processing units would produce correct signals (performance correct rate) against the accumulation of errors. Assuming that an error independently occurs with the probability $p$ in each processing unit, $R=(1-$ $p)^{8}$ for the modular network, whereas $R=(1-p)^{16}$ for the corresponding non-modular network. Clearly, the network performance deteriorates more rapidly in the non-modular network than in the modular one as the error probability $p$ increases (Fig. 7C). 
A Action-sequence-
selective neurons

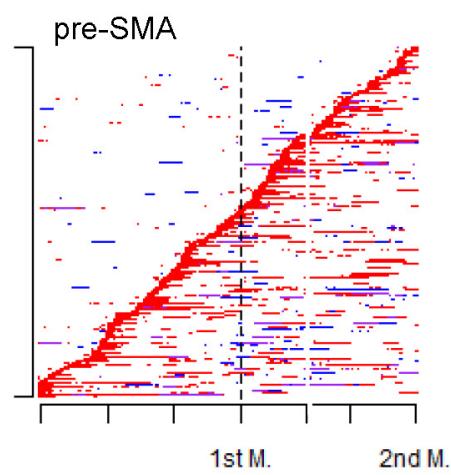

SMA

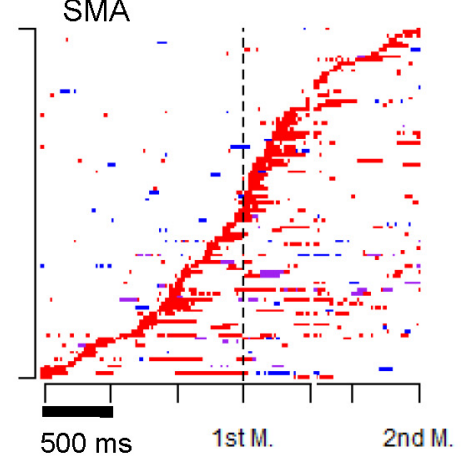

B Arm-sequence-
selective neurons

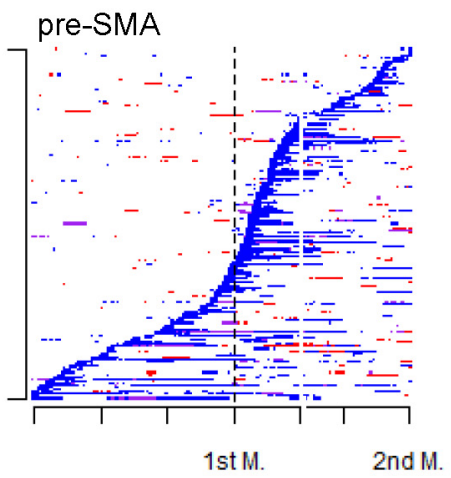

SMA

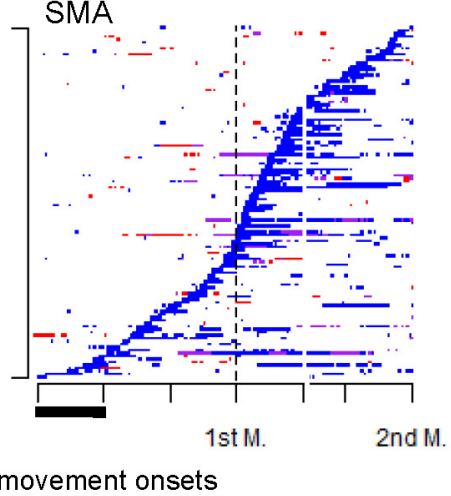

C
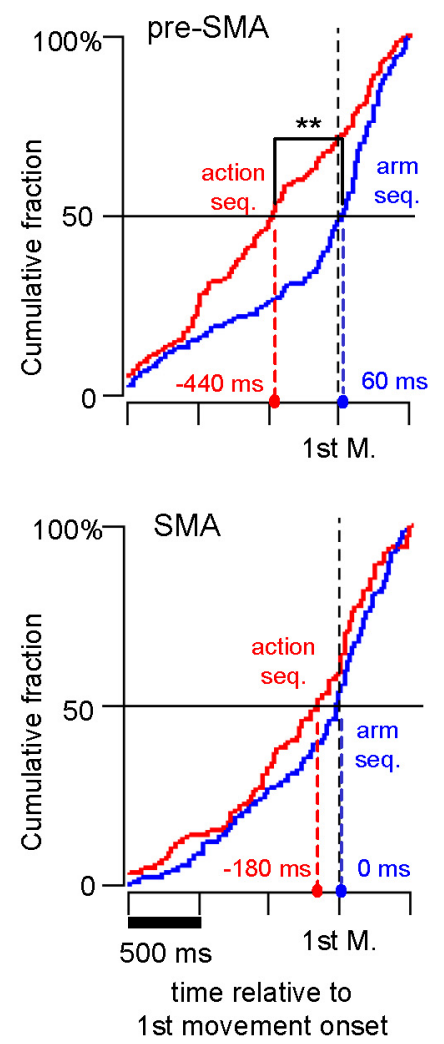

action-sequence-selective activity
arm-sequence-selective activity
movement-sequence-selective activity

Figure 6. Comparison of sequence-selective activity onset in pre-SMA and SMA neurons. $\boldsymbol{A}$, The temporal profiles of sequence-selective activity detected among action-sequence-selective neurons in the pre-SMA (top) and SMA (bottom). Sequence-selective activity, when detectable above the null model with a $200 \mathrm{~ms}$ sliding window stepped in increments of $20 \mathrm{~ms}$ across the task period, was classified into three categories: (1) action-sequence-selective activity (red); (2) arm-sequence-selective activity (blue); and (3) movement-sequence-selective activity (purple). Activity profiles of individual neurons are displayed consecutively according to color; each row represents data for a single neuron. Neuronal activity was sorted by action-sequence-selective activity onset and arranged for display in the order of its first appearance (from bottom to top). Vertical dashed lines indicate the onset time of the first movement (1st M.). For display purposes, we adjusted intertrial variation of the interval between the two movements by inserting a gap between $500 \mathrm{~ms}$ after the first movement onset and $800 \mathrm{~ms}$ before the second. $\boldsymbol{B}$, Temporal profiles of sequence-selective activity detected among arm-sequence-selective neurons. The format is the same as for $\boldsymbol{A}$, with the exception that neuronal activity is sorted by arm-sequence-selective activity onset (blue). C, Cumulative fractions of action-sequence-selective neurons (red trace) and arm-sequence-selective neurons (blue trace) are plotted during the $2 \mathrm{sinterval,} 1.5 \mathrm{~s}$ preceding, and $0.5 \mathrm{~s}$ after onset of the first movement. The horizontal axis indicates the time relative to the first movement onset (black vertical dashed line). Colored dashed lines indicate median onset times for action-sequence-selective neurons (red) and arm-sequence-selective neurons (blue). ${ }^{* *} p<0.01$ by Mann-Whitney $U$ test.

The efficiency of the two networks was compared by defining the search cost $(C)$ for each network as the expected number of searches needed to specify a particular set of processing units. This $C$ is a basis to calculate running time (or time complexity) of a search algorithm in computer science (Cormen et al., 2009). Generally, when searching for a particular unit in a list that contains $N$ units, $C$ is given as $(1+2+\ldots+N) / N=(N+1) / 2$ using a linear search (Knuth, 1998). For the non-modular network where $N=4 \times 4$ (Fig. $7 B), C=(16+1) / 2=8.5$. For the corresponding modular network (Fig. $7 A$ ), a pair of appropriate processing units should be searched from two modules. Here, $C$ is given as a pair of $(4+1) / 2$, i.e., $C=5$. To generalize this estimation in terms of the sequence length $(k)$, let us consider $k$-element motor sequences consisting of pronation or supination of either forearm. To represent all the possible $k$-element sequences with a non-modular network, $N=4^{k}$ processing units are required. Here, $C$ for the determination of a specific processing unit in the non-modular network should be given by $\left(4^{k}+1\right) / 2$. For the corresponding modular network, $C$ is given as a pair of $\left(2^{k}+\right.$ 1)/2, i.e., $C=2^{k}+1$. Apparently, the search cost increases more rapidly in the non-modular network than in the modular one as the sequence length $k$ increases (Fig. 7D). These calculations demonstrate the greater efficiency of the modular network over the non-modular one.

\section{Discussion}

We analyzed neuronal activity in the medial motor areas while monkeys performed a motor task that required temporal organization of 16 different sequences of bilateral arm movements. The primary aim was to determine whether neural encoding of as many as 16 motor sequences required group-based neuronal representation of movements with respect to common attributes in the functional or anatomical domain. Examination of the neuronal activity immediately preceding the execution of individual movements revealed that the representation of each forthcoming motor element in the task was primarily dependent on attribute 
A Two-dimensional (modular) coding

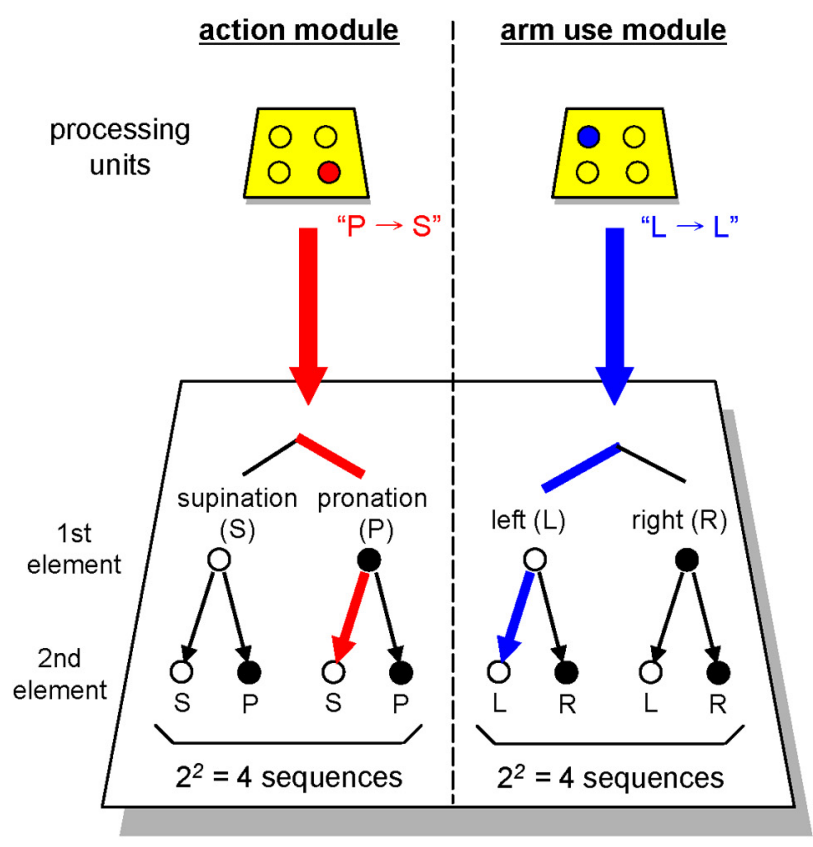

C Estimation of robustness against error probability

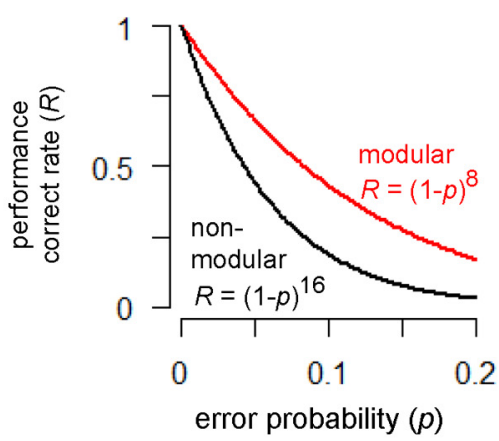

B Movement-based (non-modular) coding

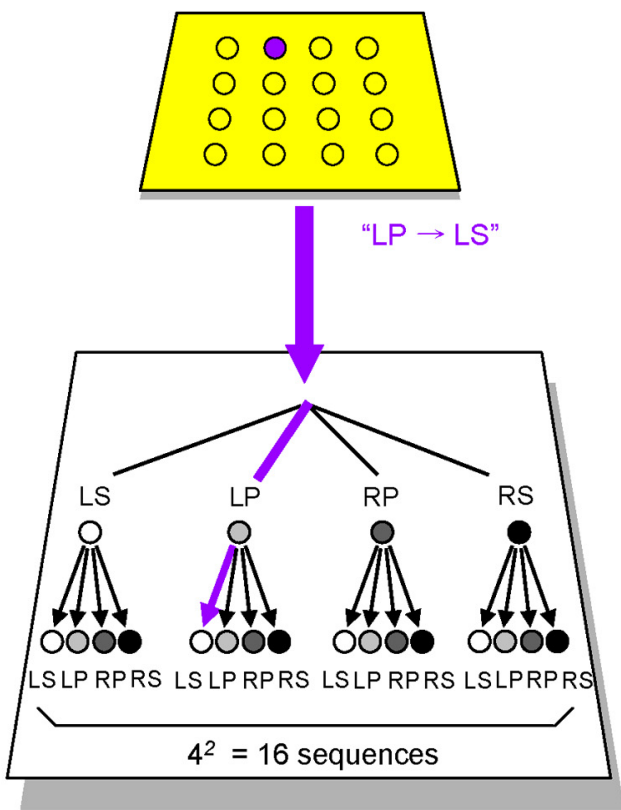
Dstimation of efficiency
for $k$-element sequence

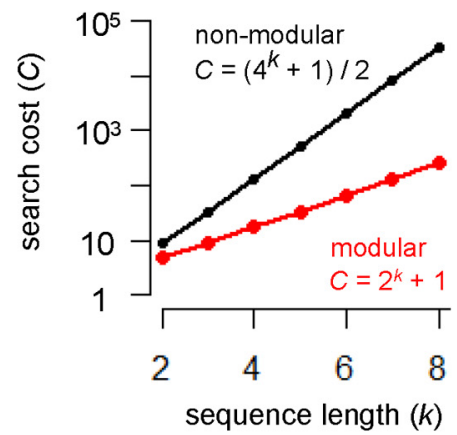

Figure 7. Schematic network models for processing multiple motor sequences involving bilateral arm actions. $A$, Neural network equipped with action module (left) and arm use module (right) processing the left pronation-left supination (LP-LS) sequence. Each circle on the yellow panels (top) represents a unit that processes a particular sequence of either action or arm use. A pair of processing units that correspond to the pronation-supination sequence (for action module) and the left arm-left arm sequence (for arm-use module) is highlighted. White or black circles (bottom) stand for neural elements that represent action or arm use occurring in a sequence. $\boldsymbol{B}$, Neural network for non-modular processing of behavioral sequences. One of the 16 processing units that corresponds to the LP-LS sequence is highlighted (top). Circles in graded colors (bottom) stand for neural elements that represent four individual movements (i.e., supination or pronation of either forearm) occurring in a sequence. $C$, Estimation of robustness $(R)$ when sequence length was two. Network performance $(R)$ was estimated by plotting the correct rate at which all the processing units in the network produced a correct signal against the probability $(p)$ of errors in each unit. The red line indicates the performance of the modular network with a total of eight processing units in the two modules, whereas the black line indicates the performance of the corresponding non-modular network that houses $4 \times 4=16$ processing units. $D$, Estimation of efficiency when the sequence length grows. Search $\operatorname{cost}(\mathrm{C})$ for a modular network (red line) and that for the non-modular network (black line) was plotted against the sequence length $(k$ ). The $C$ is plotted in logarithmic scale.

coding in terms of action (either supination or pronation) or arm use (right or left). A subsequent examination of neuronal activity representing motor sequences revealed that a significant proportion of the activity reflected action or arm-use sequences rather than individual movement sequences per se, suggesting a predominance of two-dimensional encoding of behavioral sequences in the medial motor areas.

\section{Group coding of functional/anatomical attributes and} dominance of the body-symmetric reference frame An analysis of the neuronal activity representing each motor element in the behavioral task revealed that the majority of neurons reflected the functional or anatomical attributes of individual movements. This tendency was more pronounced in the preSMA than in the SMA. Attribute coding of motor elements was observed in action-selective neurons (for functional attribute) and arm-use-selective neurons (for anatomical attribute). Action-selective neurons were more abundant in the pre-SMA than in the SMA. This finding agrees with the previous study (Fujii et al., 2002) supporting the view that the pre-SMA plays a role in effector-independent motor control. Our novel finding as to the neuronal representation of action is that the bodysymmetric reference frame dominates the object-manipulative reference frame when pronation and supination are performed 
without visuospatial cues. Previous studies looking into the preferential use of homologous muscle activation (Kelso, 1984; Kennerley et al., 2002; Carson, 2005) suggest that movements may be grouped according to the longitudinal axis of the body using a mirror-symmetric reference frame. Nevertheless, other potential reference frames cannot be ruled out; more than one reference frame can coexist, and the prevalence of one over the other may depend on the context of the task (Swinnen and Wenderoth, 2004).

In contrast, we observed more arm-use-selective neurons in the SMA than in the pre-SMA. This observation is in line with a study using an arm-target selection task (Hoshi and Tanji, 2004). The medial motor areas are closely interconnected with the motor areas in the contralateral hemisphere (Pandya and Vignolo, 1971). Thus, the coexistence of action-selective activity and armuse-selective activity may be useful to inform the contralateral hemisphere of action and arm use planned in the ipsilateral side (Brinkman, 1984; Tanji et al., 1987).

We found a small but sizeable number of neurons that were selective for individual movements, representing the effector and action elements of each movement. Such movement-selective neurons were more prevalent in the SMA than in the pre-SMA. Our results suggest that the pre-SMA is more involved in the abstract representation of motor elements than the SMA, whereas the SMA plays a greater role in more direct aspects of motor control. However, SMA neurons may not be involved substantially in specifying motor parameters (Tanji, 1996), encoding muscle activity (Alexander and Crutcher, 1990), or the timing of motor initiation (Tanji and Kurata, 1982; Scangos and Stuphorn, 2010). This view is supported by differences in the anatomical connectivity of the two areas. The SMA, but not the pre-SMA, has direct connections to the primary motor cortex and spinal cord (Dum and Strick, 1991; Luppino et al., 1993), whereas the preSMA receives afferent projections from the dorsolateral prefrontal cortex (Luppino et al., 1993; Lu et al., 1994).

\section{Two-dimensional attribute coding of motor sequences based on action and arm use}

The majority of neurons represented motor sequences with respect to functional (action) or anatomical (arm use) attributes of the individual components of the motor task. Only a small fraction of neurons represented individual movement sequences. Examination of the time of occurrence of sequence-selective activity revealed that the selectivity appeared in a wide range of the task periods. These findings are taken to indicate that neurons in the two medial motor areas take part in representing the behavioral sequences in a two-dimensional manner, with one involved in coding the action sequence and the other in coding the arm-use sequence. We noted that sequence-selective activity with regard to action emerged earlier than selectivity for arm use, despite considerable overlap. This finding is consistent with a report that suggested the role of the pre-SMA in the initiation of overlearned motor sequences or sequence chunk (Kennerley et al., 2004).

The crucial importance of the medial motor areas in the temporal organization of sequential actions has been inferred based on research in a wide range of fields (Luria, 1966; Laplane et al., 1977; Brinkman, 1984; Mushiake et al., 1991; Clower and Alexander, 1998; Grafton et al., 1998; Shima and Tanji, 2000; Berdyyeva and Olson, 2010). However, these studies did not address the way in which the nervous system processes a large number of motor sequences. A previous study addressed this problem from the perspective of categorization of motor sequences, and prefrontal neurons were shown to encode motor sequences in a category-based manner (Shima et al., 2007). In contrast, the present study dealt with even greater number of sequences involving bilateral appendages, revealing two-dimensional coding of motor sequences in the medial motor areas based on common attributes of the individual movements.

One of our novel findings as to neural encoding of motor sequences is the demonstration of the neural correlates of effector-independent motor sequencing in a nonspatial functional domain (i.e., action-sequence-selective neurons). The notion that representation of motor sequences can occur independently of effectors is supported by previous literature (Keele et al., 1995; Grafton et al., 1998; Hikosaka et al., 1999), although these reports dealt with visuospatial motor sequencing. More recently, involvement of the SMA in the intermanual transfer of motor skills was demonstrated (Perez et al., 2008). Conversely, our finding of arm-sequence-selective neurons suggests that the temporal order of effector use is regulated by the medial motor areas. This view is supported by a clinical report on patients with lesions in the medial motor areas who had difficulty in changing effector use smoothly from one hand to the other (Luria, 1966). Our findings of a substantial number of action- and armsequence-selective neurons and apparent differences in the time course of their appearance indicate that the neural mechanisms controlling behavioral sequences is internally decomposed into parallel processes (i.e., action sequencing and arm-use sequencing). Our study did not facilitate decomposition with the use of sensory instruction signals, and we believe that distinct sequencing modules are internally generated to subserve action-based and effector-based sequencing.

\section{Conclusion}

When confronted with the task of processing a large number of behavioral sequences involving bilateral use of the arms, neuronal activity in the SMA and pre-SMA reflects group-based representation of the functional (action) and anatomical (arm use) attributes of the individual movements. The behavioral sequence is primarily represented with respect to action or arm use rather than as the order of individual movements. The two-dimensional coding is viewed as producing robust and efficient processing of sequential information by neuronal networks in the medial motor areas. These findings provide the basis for understanding mechanisms of impairments in sequential behavior among patients with lesions in the medial motor area (Gentilucci et al., 2000 ) or the basal ganglia with which the medial frontal cortex is closely linked (Marsden, 1984; Graybiel, 1998).

\section{References}

Alexander GE, Crutcher MD (1990) Preparation for movement: neural representations of intended direction in three motor areas of the monkey. J Neurophysiol 64:133-150. Medline

Amador N, Fried I (2004) Single-neuron activity in the human supplementary motor area underlying preparation for action. J Neurosurg 100:250 259. CrossRef Medline

Berdyyeva TK, Olson CR (2010) Rank signals in four areas of macaque frontal cortex during selection of actions and objects in serial order. J Neurophysiol 104:141-159. CrossRef Medline

Birkhoff G (1993) Lattice theory. Providence, RI: American Mathematical Society.

Brinkman C (1984) Supplementary motor area of the monkey's cerebral cortex: short- and long-term deficits after unilateral ablation and the effects of subsequent callosal section. J Neurosci 4:918-929. Medline

Burnham KP, Anderson DR (2002) Model selection and multimodel inference: a practical information-theoretic approach. New York: Springer.

Carson RG (2005) Neural pathways mediating bilateral interactions between the upper limbs. Brain Res Rev 49:641-662. CrossRef Medline 
Clower WT, Alexander GE (1998) Movement sequence-related activity reflecting numerical order of components in supplementary and presupplementary motor areas. J Neurophysiol 80:1562-1566. Medline

Cormen TH, Leiserson CE, Rivest RL, Stein C (2009) Introduction to algorithms. Cambridge, MA: Massachusetts Institute of Technology.

Dum RP, Strick PL (1991) The origin of corticospinal projections from the premotor areas in the frontal lobe. J Neurosci 11:667-689. Medline

Fujii N, Mushiake H, Tanji J (2002) Distribution of eye- and armmovement-related neuronal activity in the SEF and in the SMA and PreSMA of monkeys. J Neurophysiol 87:2158-2166. Medline

Gentilucci M, Bertolani L, Benuzzi F, Negrotti A, Pavesi G, Gangitano M (2000) Impaired control of an action after supplementary motor area lesion: a case study. Neuropsychologia 38:1398-1404. CrossRef Medline

Gerloff C, Corwell B, Chen R, Hallett M, Cohen LG (1997) Stimulation over the human supplementary motor area interferes with the organization of future elements in complex motor sequences. Brain 120:1587-1602. Medline

Grafton ST, Hazeltine E, Ivry RB (1998) Abstract and effector-specific representations of motor sequences identified with PET. J Neurosci 18:94209428. Medline

Graybiel AM (1998) The basal ganglia and chunking of action repertoires. Neurobiol Learn Mem 70:119-136. CrossRef Medline

Hatakeyama M, Tsuda I (2007) Internal logic viewed from observation space: theory and a case study. Biosystems 90:273-286. CrossRef Medline

Hikosaka O, Nakahara H, Rand MK, Sakai K, Lu X, Nakamura K, Miyachi S, Doya K (1999) Parallel neural networks for learning sequential procedures. Trends Neurosci 22:464-471. CrossRef Medline

Hoshi E, Tanji J (2004) Differential roles of neuronal activity in the supplementary and presupplementary motor areas: from information retrieval to motor planning and execution. J Neurophysiol 92:3482-3499. CrossRef Medline

Hoshi E, Tanji J (2006) Differential involvement of neurons in the dorsal and ventral premotor cortex during processing of visual signals for action planning. J Neurophysiol 95:3596-3616. CrossRef Medline

Keele S, Jennings P, Jones S, Caulton D, Cohen A (1995) On the modularity of sequence representation. J Motor Behav 27:17-30. CrossRef

Kelso JA (1984) Phase transitions and critical behavior in human bimanual coordination. Am J Physiol 246:R1000-R1004. Medline

Kennerley SW, Diedrichsen J, Hazeltine E, Semjen A, Ivry RB (2002) Callosotomy patients exhibit temporal uncoupling during continuous bimanual movements. Nat Neurosci 5:376-381. CrossRef Medline

Kennerley SW, Sakai K, Rushworth MF (2004) Organization of action sequences and the role of the pre-SMA. J Neurophysiol 91:978-993. Medline

Knuth DE (1998) Sorting and searching. Reading, MA: Addison-Wesley.

Laplane D, Talairach J, Meininger V, Bancaud J, Orgogozo JM (1977) Clinical consequences of corticectomies involving the supplementary motor area in man. J Neurol Sci 34:301-314. CrossRef Medline

Lashley K (1951) The problem of serial order in behavior. New York: Wiley.

Louie AH (1985) Categorical system theory. In: Theoretical biology and complexity: three essays on the natural philosophy of complex systems, pp 69-163. Orlando, FL: Academic.

Lu MT, Preston JB, Strick PL (1994) Interconnections between the prefrontal cortex and the premotor areas in the frontal lobe. J Comp Neurol 341:375-392. CrossRef Medline

Luppino G, Matelli M, Camarda R, Rizzolatti G (1993) Corticocortical connections of area F3 (SMA-proper) and area F6 (pre-SMA) in the macaque monkey. J Comp Neurol 338:114-140. CrossRef Medline
Luria A (1966) Higher cortical functions in man. London: Tavistock.

Marsden CD (1984) Motor disorders in basal ganglia disease. Hum Neurobiol 2:245-250. Medline

Matsuzaka Y, Aizawa H, Tanji J (1992) A motor area rostral to the supplementary motor area (presupplementary motor area) in the monkey: neuronal activity during a learned motor task. J Neurophysiol 68:653-662. Medline

Mushiake H, Inase M, Tanji J (1991) Neuronal activity in the primate premotor, supplementary, and precentral motor cortex during visually guided and internally determined sequential movements. J Neurophysiol 66:705-718. Medline

Pandya DN, Vignolo LA (1971) Intra- and interhemispheric projections of the precentral, premotor and arcuate areas in the rhesus monkey. Brain Res 26:217-233. CrossRef Medline

Perez MA, Tanaka S, Wise SP, Willingham DT, Cohen LG (2008) Timespecific contribution of the supplementary motor area to intermanual transfer of procedural knowledge. J Neurosci 28:9664-9669. CrossRef Medline

Raftery AE (1995) Bayesian model selection in social research. Sociol Methodol 25:111-163. CrossRef

Rieke F (1996) Spikes: exploring the neural code. Cambridge, MA: Massachusetts Institute of Technology.

Rizzolatti G, Fogassi L, Gallese V (2001) Neurophysiological mechanisms underlying the understanding and imitation of action. Nat Rev Neurosci 2:661-670. CrossRef Medline

Rosenbaum D (2010) Human motor control, Ed 2. Amsterdam: Elsevier.

Sakamoto Y, Ishiguro M, Kitagawa G (1986) Analysis of variance models. In: Akaike information criterion statistics. Dordrecht, The Netherlands: Reidel.

Scangos KW, Stuphorn V (2010) Medial frontal cortex motivates but does not control movement initiation in the countermanding task. J Neurosci 30:1968-1982. CrossRef Medline

Shima K, Tanji J (2000) Neuronal activity in the supplementary and presupplementary motor areas for temporal organization of multiple movements. J Neurophysiol 84:2148-2160. Medline

Shima K, Isoda M, Mushiake H, Tanji J (2007) Categorization of behavioural sequences in the prefrontal cortex. Nature 445:315-318. CrossRef Medline

Simoncelli EP, Olshausen BA (2001) Natural image statistics and neural representation. Annu Rev Neurosci 24:1193-1216. CrossRef Medline

Sreenivasan S, Fiete I (2011) Grid cells generate an analog error-correcting code for singularly precise neural computation. Nat Neurosci 14:13301337. CrossRef Medline

Swinnen SP, Wenderoth N (2004) Two hands, one brain: cognitive neuroscience of bimanual skill. Trends Cogn Sci 8:18-25. CrossRef Medline

Tanji J (1996) New concepts of the supplementary motor area. Curr Opin Neurobiol 6:782-787. CrossRef Medline

Tanji J (2001) Sequential organization of multiple movements: involvement of cortical motor areas. Annu Rev Neurosci 24:631-651. CrossRef Medline

Tanji J, Kurata K (1982) Comparison of movement-related activity in two cortical motor areas of primates. J Neurophysiol 48:633-653. Medline

Tanji J, Okano K, Sato KC (1987) Relation of neurons in the nonprimary motor cortex to bilateral hand movement. Nature 327:618-620. CrossRef Medline

Zar JH (2010) Biostatistical analysis. Upper Saddle River, NJ: Prentice-Hall/ Pearson. 